\title{
Long-Term Clinical Outcomes of Invasive Giant Prolactinomas after a Mean Ten-Year Followup
}

\author{
Ze Rui Wu, ${ }^{1}$ Yong Zhang, ${ }^{1}$ Lin Cai, ${ }^{1}$ Shao Jian Lin, ${ }^{2}$ Zhi Peng Su, ${ }^{1}$ Yong Xu Wei, ${ }^{2}$ \\ Han Bing Shang, ${ }^{2}$ Wen Lei Yang, ${ }^{2}$ Wei Guo Zhao, ${ }^{2}$ and Zhe Bao Wu ${ }^{1,2}$ \\ ${ }^{1}$ Department of Neurosurgery, First Affiliated Hospital of Wenzhou Medical University, Wenzhou 325000, China \\ ${ }^{2}$ Department of Neurosurgery, Ruijin Hospital, Shanghai Jiao Tong University School of Medicine, Shanghai 200025, China
}

Correspondence should be addressed to Zhe Bao Wu; zhebaowu@aliyun.com

Received 10 July 2016; Accepted 24 October 2016

Academic Editor: Aldo E. Calogero

Copyright (C) 2016 Ze Rui Wu et al. This is an open access article distributed under the Creative Commons Attribution License, which permits unrestricted use, distribution, and reproduction in any medium, provided the original work is properly cited.

Objective. The aim of this study is to observe clinical outcomes after more than ten years of followup in a group of patients with invasive giant prolactinomas (IGPs) treated with dopamine agonists (DAs). Methods. Twenty-five patients met the criteria of IGPs, among which 16 patients primarily received bromocriptine (BRC) and the other nine had undergone unsuccessful microsurgery prior to BRC treatment. Results. After a mean follow-up period of $135.5 \pm 4.7$ months, the clinical symptoms in all patients improved by different degrees. Tumor volume was decreased by a mean of $98.6 \%$, and the tumors of 19 patients had almost completely disappeared. The mean duration of treatment at maximal doses of BRC was 48.5 months. At the last follow-up visit, nineteen patients had normal PRL levels, and 14 of these patients had received the low-dose BRC treatment (at an average of $2.9 \pm 0.3 \mathrm{mg} / \mathrm{d}$ ). Younger patients $<25$ years had a significantly higher rate of persistent hyperprolactinemia after long-term BRC treatment $(p=0.043)$. Conclusion. DAs are a first-line therapy for IGPs because they can effectively achieve long-term control in both shrinking tumor volume and normalizing the PRL level, and majority of patients need low-dose DA maintenance. Younger patients are prone to persistent hyperprolactinemia despite long-term DA treatment.

\section{Introduction}

Prolactinoma is the most common type of pituitary adenoma. Dopamine agonists (DAs), such as bromocriptine (BRC) and cabergoline $(\mathrm{CAB})$, are the first-line treatment and effectively suppress prolactin (PRL) hypersecretion, reduce tumor size, and restore gonadal function [1-6]. DA's effect is not only positively correlated with dopamine 2 receptor (D2R) but also related to the expression ratio of the two isoforms of D2R on the surface of prolactinoma cells $[7,8]$. Invasive giant prolactinomas (IGPs) are a rare subtype of prolactinomas whose criteria include a maximum tumor diameter greater than $40 \mathrm{~mm}$, a serum prolactin concentration higher than $200 \mathrm{ng} / \mathrm{mL}$, and tumor invasion into the cavernous sinus to an extent corresponding to Grade III or IV according to the classification scheme of Knosp and colleagues [9]. IGPs have an incidence rate of $0.5 \%-4.4 \%$ in pituitary adenomas $[10,11]$. Because of its aggressive clinical behavior, the biochemical and tumor volume control in this subtype is a significant challenge [12-14].
There are a few data regarding the treatment outcomes of IGPs; however, large series are limited, and evidence-based recommendations of DAs are therefore lacking, especially for long-term treatment strategy. We previously reported 20 patients with IGPs who were treated with BRC and had a mean follow-up period of 37.3 months; we found that BRC was effective as a first-line therapy for this subtype of prolactinomas, with a mean tumor volume decrease of 93.3\%. However, clinical outcomes after more than ten years of followup are largely unknown for DA-treated IGPs. In our study, we investigate the clinical outcomes after more than ten years of followup in a group of patients with IGPs treated with DAs.

\section{Methods}

2.1. Patient Population and Inclusion Criteria. Consistent with our previous report [2], the criteria to qualify for study participation included (1) a maximum tumor diameter 
greater than $4 \mathrm{~cm}$ invading the cavernous sinus to an extent corresponding to Grade III or IV, according to the classification scheme of Knosp and colleagues [9]; (2) a serum PRL level greater than $200 \mathrm{ng} / \mathrm{mL}$ [2]; and (3) clinical signs of hyperprolactinemia and mass effect. If patients underwent microsurgery before DAs treatment, these patients met the diagnostic criteria for IGPs according to postoperative MR imaging and endocrinological examination data and were therefore included in our study. To determine the outcomes of DAs treatment, tumor volume was calculated based on postoperative MR images in the same manner as the volume before DAs treatment.

From August 1998 to September 2007, twenty-five consecutive patients with IGPs invading the cavernous sinus were treated at our hospitals. In our previous report of 20 patients with IGPs, eight patients were lost to followup and were thus excluded from the study [2]. Seven patients were also reported in our other previous studies [13, 15]. This study was approved by the Ethics Committee of Shanghai Jiao Tong University School of Medicine and Wenzhou Medical University, and written informed consent was obtained from all patients. The cohort consisted of 18 male and 7 female patients, whose ages ranged from 15 to 52 years (mean age $35.7 \pm 2.2$ years) and whose pretreatment course of the disease persisted from 69 months to 192 months (mean course 102.5 months). The detailed information regarding patients' clinical characteristics is listed in Table 1.

\subsection{Clinical Manifestation. A visual deficit was a common} symptom with an incidence of $52 \%$ ( 13 of 25 patients), as well as headache with an incidence of $40 \%$ (10 of 25 patients). Amenorrhea was the initial manifestation in 5 of 7 women (71\%), and decreased libido was the presenting symptom in 13 men ( $72 \%$ of men in our cohort). The most frequent symptoms and signs are detailed in Table 2.

\subsection{Neuroradiological and Endocrinological Evaluation.} Tumor mass was evaluated with a series of MR images as previously reported [2]. The maximal tumor diameter was calculated in millimeters, and tumor volume was calculated using the following formula: $V=\pi a b c / 6$ where $a, b$, and $c$ are the three diameters of the tumor. According to these baseline MR images, maximal tumor diameters ranged from 40 to $73 \mathrm{~mm}$. The tumor sizes and invaded parasellar areas are listed in Table 1. Invasion of the bilateral cavernous sinuses occurred in 13 cases, and invasion of a unilateral cavernous sinus occurred in the other 12 cases.

All PRL levels were greater than $200 \mathrm{ng} / \mathrm{mL}$ before BRC treatment and exceeded $4000 \mathrm{ng} / \mathrm{mL}$ in nine cases. Due to difficulties in the laboratory, accurate PRL levels were not available in some cases. Recurrence of hyperprolactinaemia following discontinuation of BRC therapy was defined as a serum PRL level greater than the sex-specific upper limit of the normal range for the assay used [serum PRL $>16 \mathrm{ng} / \mathrm{mL}$ (men) and $>25 \mathrm{ng} / \mathrm{mL}$ (women)].

2.4. Treatment Protocol. From this cohort, 16 patients were primarily treated with BRC, and the other nine had previously undergone microsurgery with partial tumor resection prior to BRC treatment. Six of the patients underwent adjuvant radiotherapy, and two underwent gamma knife radiotherapy.

The BRC treatment protocol was followed according to our previous methods [2]: BRC was orally administered before sleep at night at an initial dose of $2.5 \mathrm{mg} /$ day and gradually increased to $7.5 \mathrm{mg} /$ day within 2 to 3 weeks, which was defined as the effective treatment dose in our study. If PRL level was not normalized, the dose would be gradually increased to $15 \mathrm{mg}$ /day and further increased dose was not recommended. In cases in which patients experienced adverse effects after taking BRC, only half of a tablet $(1.25 \mathrm{mg})$ was subsequently administered, and the dose was slowly increased. A change to $\mathrm{CAB}$ treatment was made in one patient.

2.5. Statistical Analysis. All statistical analyses were carried out using the SPSS (Statistical Package for the Social Sciences) 17.0 statistical software package. Continuous variables were expressed as the mean \pm SEM. Mean values were compared using unpaired $t$-tests, and categorical variables were compared using Fisher's exact test. The significance of various variables for persistent hyperprolactinemia was analysed using the Cox proportional hazards model in the univariate and multivariate analysis to identify which factors were independent indicators for persistent hyperprolactinemia. A two-tailed $p$ value test was used with a $p$ value of $<0.05$, considered statistically significant.

\section{Results}

3.1. Treatment Courses. All patients were closely followed up between 90 and 192 months after treatment (mean followup of $135.5 \pm 4.7$ months). Initially, two patients experienced different degrees of BRC-related side effects. A lower BRC dose was administered and gradually increased. Both patients were able to tolerate the entire dose following this strategy. Over the course of BRC administration, cerebrospinal fluid (CSF) leakage occurred in one patient (case no. 20), who subsequently underwent transsphenoidal surgery to repair the CSF leak. No cases of apoplexy occurred during BRC treatment. Panhypopituitarism had occurred in 5 patients at the last follow-up visit. No cases of diabetes insipidus were observed.

3.2. Effects of Treatment on Presenting Symptoms. With longterm DAs therapy, headache improved in 9 patients, depending on the extent of tumor shrinkage. Among the 13 patients with decreased libido, ten showed a clinical improvement in both libido and potency associated with the normalization of PRL, gonadotrophin, and testosterone levels. Among the 13 patients who presented with visual deficits, nine showed an improvement and four did not show signs of deterioration during the long-term followup. Galactorrhoea disappeared in the two patients who had presented with it. Amenorrhea returned to normal in two of the five female patients. 


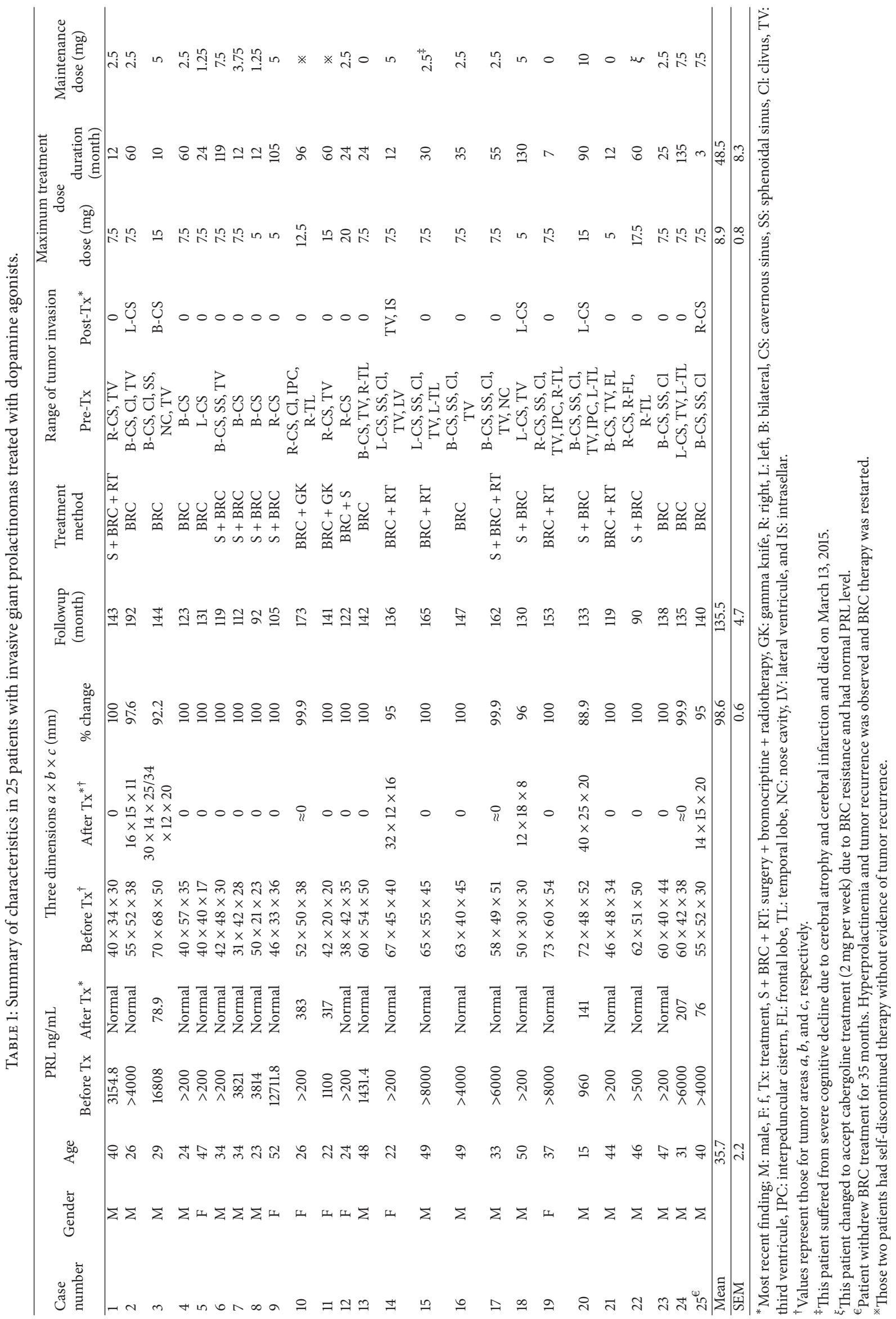


TABLE 2: Pretreatment manifestations in 25 patients with IGPs.

\begin{tabular}{lcc}
\hline Pretreatment presentations & Patient number & \% of patients \\
\hline Visual change & 13 & 52 \\
Headache & 10 & 40 \\
Dizziness & 1 & 4 \\
Hypopituitarism & 2 & 8 \\
Cognitive decline & 1 & 4 \\
Decreased sexual function $^{\dagger}$ & 13 & 72 \\
Amenorrhea $^{\ddagger}$ & 5 & 71 \\
Galactorrhea $^{\ddagger}$ & 2 & 29 \\
Nasal obstruction and hemorrhage $^{\text {Axillary and pubic hair loss }}$ & 2 & 8 \\
\hline
\end{tabular}

${ }^{\dagger}$ In males, the decreased sexual function was 13/18.

${ }^{\ddagger}$ In females, the incidence of amenorrhea and galactorrhea was 5/7 and 2/7, respectively.

3.3. Effects of Treatment on Tumor Volume. After more than ten years of long-term treatment with DAs, the tumor volume decreased dramatically in all patients, with a mean reduction of $98.6 \pm 0.6 \%$ (range from $88.9 \%$ to $100 \%$, Table 1 ). The tumor had almost completely disappeared in 19 patients; for the other six patients, residual tumor remained in the cavernous sinus area in five cases. The mean duration of treatment at maximal doses of DA $(8.9 \pm 0.8 \mathrm{mg}$ per day) was $48.5 \pm 8.3$ months (range from 3 months to 135 months).

3.4. Effects of Treatment on PRL Level. Resistance to BRC was defined by an absence in the normalization of PRL levels when administered in a daily dose of $15 \mathrm{mg}$ for at least 3 months $[8,16,17]$. In our series, five patients were resistant to BRC treatment (cases number 3, number 11, number 12, number 20, and number 22). Patient number 12 accepted microsurgery due to BRC resistance and finally had normal PRL level. Patient number 22 changed to accept cabergoline treatment due to BRC resistance and had normal PRL level. In addition, one patient (case number 10) received high dose BRC treatment (12.5 mg/d) for 96 months, but her hyperprolactinemia was not normalized; she was referred to as the resistant case. The incidence of resistance was $24 \%$ $(6 / 25)$ in our study.

At the end of followup, 20 patients were still on BRC treatment, three withdrew from BRC, and two self-discontinued BRC. Nineteen patients had normal PRL levels (the rate of PRL normalization was 76\%), as shown in Table 1; one of these patients changed to $\mathrm{CAB}$ treatment $(2 \mathrm{mg} / \mathrm{wk}$, case number 22), one accepted effective doses of BRC treatment $(7.5 \mathrm{mg} / \mathrm{d}$, case number 6), three withdrew from BRC (cases number 13 , number 19 , and number 21 ), and the other 14 patients accepted the low-dose BRC treatment $(\leq 5 \mathrm{mg} / \mathrm{d}$, at an average of $2.9 \pm 0.3 \mathrm{mg} / \mathrm{d}$ ). BRC dose $\leq 2.5 \mathrm{mg} / \mathrm{d}$ was maintained in 10 patients. Three patients with residual tumors who received low-dose BRC treatment (cases number 2, number 14, and number 18) had normal PRL levels without clinical signs of hyperprolactinemia or mass effect.

In six patients, PRL levels exceeded $25 \mathrm{ng} / \mathrm{mL}, 3$ of whom had more than $200 \mathrm{ng} / \mathrm{mL}$ at the last follow-up visit. Among them, four patients (cases number 3, number 10, number 11 , and number 20) were resistant to BRC treatment. Two patients (case number 10 and number 11) received gamma knife radiotherapy due to having a persistent residual tumor in the cavernous sinus area. Although the tumor volume almost disappeared, the PRL levels continued to exceed $200 \mathrm{ng} / \mathrm{mL}$ during the long-term followup period. These two patients self-discontinued BRC treatment; however, no obvious evidence of tumor recurrence was observed. Patient number 24 received BRC treatment with a dose of $7.5 \mathrm{mg} /$ day for 135 months; however, PRL level did not reach the normal range. Patient number 25 was initially very sensitive to BRC treatment, and the PRL level decreased to the normal range; after BRC withdrawal, the patient experienced hyperprolactinemia and tumor recurrence, and BRC therapy was readministered.

3.5. Predictor of Persistent Hyperprolactinemia. According to whether patients had persistent hyperprolactinemia during long-term DA treatment, patients were divided into two groups: the normal PRL group and the persistent hyperprolactinemia group. Patient number 25 was initially sensitive to BRC treatment with the normalization of PRL level, so this patient was not included in the persistent hyperprolactinemia group. Thus, the persistent hyperprolactinemia group contained five patients (cases numbers $3,10,11,20$, and 24).

The relationship between clinicopathologic features and persistent hyperprolactinemia in 25 patients is summarized in Table 3. There were no statistically significant associations between persistent hyperprolactinemia and gender, tumor volume before BRC treatment, final tumor volume, tumor reduction percentage, unilateral or bilateral cavernous sinus invasion, duration of followup, treatment method, or duration of maximum BRC dose. A significant correlation was observed between persistent hyperprolactinemia and age at diagnosis $(p=0.009)$, or maximum BRC dose $(p=$ $0.015)$. To determine whether age at diagnosis was important prognostic factor for persistent hyperprolactinemia among those clinicopathologic features, univariate Cox regression analysis was used in this 25-patient cohort, which indicated that age at diagnosis was important prognostic factors for persistent hyperprolactinemia $(p=0.043$, Table 4). Furthermore, multivariate Cox regression analysis indicated that age at diagnosis was the best predictor of persistent hyperprolactinemia ( $p=0.043$, Table 4$)$.

Together, the results showed that age at diagnosis was the only independent prognostic factor of persistent hyperprolactinemia, and it indicated that younger patients were prone to resistance to long-term BRC treatment.

\section{Discussion}

Dopamine agonists have been demonstrated as a first-line therapy for IGPs, because they can significantly shrink tumor volume and control the PRL levels [2, 10-12, 14, 18-25]. As for giant prolactinomas, the overall tumor response rate to DA treatment was $47 \%-87 \%[10,11,14,19,20,22,25]$. In Maiter and Delgrange's recent review [14], the overall tumor 
TABLE 3: Relations between PRL levels and clinicopathologic features in IGPs.

\begin{tabular}{|c|c|c|c|}
\hline \multirow{2}{*}{ Clinicopathologic parameters } & \multicolumn{2}{|c|}{ PRL levels } & \multirow{2}{*}{$p$ value } \\
\hline & Normal $(n=20)$ & Hyperprolactinemia $(n=5)$ & \\
\hline Age & $38.4 \pm 2.3$ & $24.6 \pm 2.8$ & 0.009 \\
\hline Gender & & & 0.597 \\
\hline Male & 15 & 3 & \\
\hline Female & 5 & 2 & \\
\hline Tumor volume before treatment & $3.18 \pm 0.4 \times 10^{6}$ & $4.2 \pm 1.3 \times 10^{6}$ & 0.33 \\
\hline Final tumor volume & & & 0.562 \\
\hline No remnant & 16 & 3 & \\
\hline Remnant & 4 & 2 & \\
\hline Tumor reduction percentage $\%$ & $99.2 \pm 0.39$ & $96.2 \pm 2.4$ & 0.275 \\
\hline Cavernous sinus invasion & & & 0.645 \\
\hline Bilateral & 11 & 2 & \\
\hline Unilateral & 9 & 3 & \\
\hline Duration of followup & $133.05 \pm 5.5$ & $145.23 \pm 7.2$ & 0.311 \\
\hline Treatment strategy & & & 0.621 \\
\hline BRC & 12 & 4 & \\
\hline $\mathrm{S}+\mathrm{BRC}^{\oint}$ & 8 & 1 & \\
\hline Maximum BRC dose & $8.1 \pm 0.85$ & $13 \pm 1.45$ & 0.015 \\
\hline Duration of maximum BRC dose & $41.0 \pm 8.5$ & $78.2 \pm 20.8$ & 0.074 \\
\hline
\end{tabular}

TABLE 4: Summary of persistent hyperprolactinemia analyses by univariate and multivariate COX regression analysis.

\begin{tabular}{|c|c|c|c|c|}
\hline \multirow{2}{*}{ Variable } & \multicolumn{2}{|c|}{ Univariate analysis } & \multicolumn{2}{|c|}{ Multivariate analysis $^{\&}$} \\
\hline & $\operatorname{HR}(95 \% \mathrm{CI})$ & $p$ value & $\operatorname{HR}(95 \% \mathrm{CI})$ & $p$ value \\
\hline Age $(\geq 25$ y versus $<25$ y) & $0.082(0.007-0.929)$ & 0.043 & & \\
\hline Gender (male versus female) & $0.723(0.12-4.65)$ & 0.727 & & \\
\hline Final tumor volume (no remnant versus remnant) & $1.434(0.23-8.97)$ & 0.7 & $0.082(0.007-0.929)$ & 0.043 \\
\hline Cavernous sinus invasion (bilateral versus unilateral) & $0.612(0.10-3.67)$ & 0.59 & & \\
\hline Treatment strategy $\left(\mathrm{BRC}\right.$ versus $\left.\mathrm{S}+\mathrm{BRC}^{\oint}\right)$ & $1.6(0.17-15.67)$ & 0.679 & & \\
\hline
\end{tabular}

${ }^{\&}$ Probability for stepwise: entry $=0.05$ and removal $=0.1$.

$\oint_{\mathrm{S}}+\mathrm{BRC}$ : surgery + bromocriptine.

response rate for giant prolactinomas to DA treatment was $74 \%$ (65/88 evaluable cases), and the hormonal response rate was $60 \%$ (58/97 evaluable cases), with a mean followup of 37 months. Chattopadhyay et al. [22] reported that 14 patients with male giant prolactinomas weretreated with BRC, and under a maximal average BRC dose of $12.3 \mathrm{mg} /$ day with a treatment duration of 32.8 months, the tumor volume obviously shrank from 48.4 to $14.9 \mathrm{~mm}^{3}$ (69.5\%). Shrivastava and colleagues reported that in 10 cases of giant prolactinomas treated with BRC, and the tumor volume decreased by a mean of $69 \%$ during a mean follow-up period of 6.7 years [10]. In contrast, the superiority of cabergoline in terms of patient tolerability and convenience, reduction in prolactin secretion, restoration of gonadal function, and decrease in tumor volume has been convincingly demonstrated [11, 19, 20]. Acharya et al. reported ten giant prolactinomas treated with $\mathrm{CAB}$; the maximal tumor diameter progressively decreased by a mean of $49 \%$, and 7 patients achieved PRL normalization
[19]. In a study by Corsello et al., CAB caused a significant decrease in both PRL levels and tumor volume $(98.8 \%$ and $64.8 \%$, resp.) in ten male patients with IGPs during a mean of 38.9 months of followup [11]. Shimon et al. reported that, in twelve male patients with giant prolactinomas following $\mathrm{CAB}$ treatment, the tumor diameter showed a mean maximal decrease of $47 \%$ [20]. Recently, Espinosa et al. reported 47 patients with giant prolactinomas, in which $68 \%$ cases had the normalization of PRL levels and $87 \%$ had the reduction of $>50 \%$ in tumor volume after CAB treatment [25].

Obviously, these studies were conducted with a limited follow-up period. To the best of our knowledge, there are two reports that consider more than ten-year long-term clinical outcomes of giant prolactinomas. Fraioli et al. utilized multidisciplinary treatment for IGPs in two children and finally reached satisfactory control of the disease with a follow-up period of 13 and 14 years [26]. Fernandes et al. reported a giant prolactinoma with 10 years of DA treatment, 
whose prolactin levels decreased by $96.8 \%$ with an effective reduction in tumor size [27]. In our study, tumor volume shrank by a mean of $98.6 \%$ and the incidence of PRL normalization was $76 \%$. The clinical outcomes of our study are better than other references, which may be explained by the longer followup and by combination treatment with surgery and/or radiotherapy. It has been reported that surgery allows a reduction of the DA dose required to normalize PRL $[28,29]$. In addition, radiotherapy may have contributed to the long-term control of these 8 patients in our series [30].

In our study, age at diagnosis was the only independent prognostic factor of long-term persistent hyperprolactinemia, and younger patients with IGPs were prone to be resistant to DA treatment. It has been demonstrated that DA-resistant patients tend to be younger males with larger or more invasive adenomas [31-33]. A recent study on macroprolactinomas in children and adolescents indicated that DA resistance is associated with higher PRL levels and larger tumor sizes, which are closely linked together [34]. In our study, there was no significant difference in tumor volume at diagnosis between younger age $(<25)$ and older age $(\geq 25)(p=0.342)$. One of the shortfalls of this study is that accurate PRL levels are not available in some cases before BRC treatment; thus, we do not know whether pretreatment PRL level is associated with persistent hyperprolactinemia despite long-term DA therapy. On the other hand, $\mathrm{CAB}$ is not available in the mainland of China; thus, as for BRC-resistant patients, just one patient changed to $\mathrm{CAB}$ treatment, which might affect the long-term clinical outcomes.

Once the PRL level and tumor volume were adequately controlled, the dose of DA could often be decreased incrementally and then kept at a minimal maintenance dose [35]. In our study, nineteen patients had normal PRL levels and 14 of these patients accepted the low-dose BRC treatment, with an average dose of $2.9 \mathrm{mg} / \mathrm{d}$. The majority of 14 patients (10/14, $71 \%$ ) needed only a $\leq 2.5 \mathrm{mg} /$ day low-dose BRC maintenance. Additionally, three patients with residual tumors without clinical signs of mass effect also received low-dose BRC treatment to maintain PRL normalization. Together, in our study, $56 \%$ of the patients (14/25) received low-dose BRC treatments without relapse of tumor volume or PRL level.

\section{Conclusions}

In summary, we report the clinical outcomes of more than ten years of followup in a group of 25 patients with IGPs treated with BRC. DAs as first-line therapy can effectively achieve long-term control in both shrinking tumor volume and normalizing PRL levels. Additionally, 56\% of patients just need low-dose BRC treatment to maintain the long-term normalization of PRL levels and to prevent tumor recurrence. Younger patients seem to have a poor response to BRC treatment and are prone to persistent hyperprolactinemia despite long-term BRC treatment.

\section{Competing Interests}

The authors declare that they have no competing interests.

\section{Authors' Contributions}

Ze Rui Wu, Yong Zhang, and Lin Cai contributed equally to this work.

\section{Acknowledgments}

The authors are grateful to Prof. Edward Laws (Department of Neurosurgery, Brigham \& Women's Hospital, Boston) for his critical evaluation and linguistic revision of this manuscript. This work was supported by the National Natural Science Foundation of China (81271523 and 81471392) and by the Zhejiang Open Foundation of the Top Key Discipline (LKYJ015 to Zhe Bao Wu).

\section{References}

[1] M. P. Gillam, M. E. Molitch, G. Lombardi, and A. Colao, "Advances in the treatment of prolactinomas" Endocrine Reviews, vol. 27, no. 5, pp. 485-534, 2006.

[2] B. W. Zhe, J. Y. Chun, P. S. Zhi, C. Z. Qi, S. W. Jin, and M. Z. Wei, "Bromocriptine treatment of invasive giant prolactinomas involving the cavernous sinus: results of a long-term follow up," Journal of Neurosurgery, vol. 104, no. 1, pp. 54-61, 2006.

[3] F. F. Casanueva, M. E. Molitch, J. A. Schlechte et al., "Guidelines of the Pituitary Society for the diagnosis and management of prolactinomas," Clinical Endocrinology, vol. 65, no. 2, pp. 265273, 2006.

[4] S. Melmed, F. F. Casanueva, A. R. Hoffman et al., "Diagnosis and treatment of hyperprolactinemia: an endocrine society clinical practice guideline," Journal of Clinical Endocrinology and Metabolism, vol. 96, no. 2, pp. 273-288, 2011.

[5] A. Colao and S. Savastano, "Medical treatment of prolactinomas," Nature Reviews Endocrinology, vol. 7, no. 5, pp. 267-278, 2011.

[6] J. S. Bevan, J. Webster, C. W. Burke, and M. F. Scanlon, "Dopamine agonists and pituitary tumor shrinkage," Endocrine Reviews, vol. 13, no. 2, pp. 220-240, 1992.

[7] L. Caccavelli, F. Feron, I. Morange et al., "Decreased expression of the two D2 dopamine receptor isoforms in bromocriptineresistant prolactinomas," Neuroendocrinology, vol. 60, no. 3, pp. 314-322, 1994.

[8] Z. B. Wu, W. M. Zheng, Z. P. Su et al., "Expression of D2RmRNA isoforms and ERmRNA isoforms in prolactinomas: correlation with the response to bromocriptine and with tumor biological behavior," Journal of Neuro-Oncology, vol. 99, no. 1, pp. 25-32, 2010.

[9] E. Knosp, E. Steiner, K. Kitz et al., "Pituitary adenomas with invasion of the cavernous sinus space: a magnetic resonance imaging classification compared with surgical findings," Neurosurgery, vol. 33, no. 4, pp. 610-618, 1993.

[10] R. K. Shrivastava, M. S. Arginteanu, W. A. King, and K. D. Post, "Giant prolactinomas: clinical management and longterm follow up," Journal of Neurosurgery, vol. 97, no. 2, pp. 299306, 2002.

[11] S. M. Corsello, G. Ubertini, M. Altomare et al., "Giant prolactinomas in men: efficacy of cabergoline treatment," Clinical Endocrinology, vol. 58, no. 5, pp. 662-670, 2003.

[12] A. B. Moraes, C. Marques Dos Santos Silva, L. Vieira Neto, and M. R. Gadelha, "Giant prolactinomas: the therapeutic 
approach," Clinical Endocrinology, vol. 79, no. 4, pp. 447-456, 2013.

[13] Z. B. Wu, Z. P. Su, J. S. Wu, W. M. Zheng, Q. C. Zhuge, and M. Zhong, "Five years follow-up of invasive prolactinomas with special reference to the control of cavernous sinus invasion," Pituitary, vol. 11, no. 1, pp. 63-70, 2008.

[14] D. Maiter and E. Delgrange, "Therapy of endocrine disease: the challenges in managing giant prolactinomas," European Journal of Endocrinology, vol. 170, no. 6, pp. R213-R227, 2014.

[15] X. H. Lou, Z. B. Wu, and Y. Z. Zhang, "Bromocriptine-induced brainstem angulation in a patient with invasive prolactinoma," Journal of Clinical Endocrinology and Metabolism, vol. 98, no. 3, pp. 867-868, 2013.

[16] A. Di Sarno, M. L. Landi, P. Cappabianca et al., "Resistance to cabergoline as compared with bromocriptine in hyperprolactinemia: prevalence, clinical definition, and therapeutic strategy," Journal of Clinical Endocrinology and Metabolism, vol. 86, no. 11, pp. 5256-5261, 2001.

[17] A. Colao, A. Di Sarno, F. Sarnacchiaro et al., "Prolactinomas resistant to standard dopamine agonists respond to chronic cabergoline treatment," Journal of Clinical Endocrinology and Metabolism, vol. 82, no. 3, pp. 876-883, 1997.

[18] M. H. Almalki, B. Buhary, S. Alzahrani et al., "Giant prolactinomas: clinical manifestations and outcomes of 16 Arab cases," Pituitary, vol. 18, no. 3, pp. 405-409, 2015.

[19] S. V. Acharya, R. A. Gopal, P. S. Menon, T. R. Bandgar, and N. S. Shah, "Giant prolactinoma and effectiveness of medical management," Endocrine Practice, vol. 16, no. 1, pp. 42-46, 2010.

[20] I. Shimon, C. Benbassat, and M. Hadani, "Effectiveness of longterm cabergoline treatment for giant prolactinoma: study of 12 men," European Journal of Endocrinology, vol. 156, no. 2, pp. 225-231, 2007.

[21] J. R. E. Davis, M. C. Sheppard, and D. A. Heath, "Giant invasive prolactinoma: a case report and review of nine further cases," Quarterly Journal of Medicine, vol. 74, no. 275, pp. 227-238, 1990.

[22] A. Chattopadhyay, A. Bhansali, and S. R. Masoodi, "Long-term efficacy of bromocriptine in macroprolactinomas and giant prolactinomas in men," Pituitary, vol. 8, no. 2, pp. 147-154, 2005.

[23] C. M. Barrera, A. E. Ruiz, and W. A. Banks, "A giant prolactinoma and the effect of chronic bromocriptine therapy on basal and TRH-stimulated serum prolactin levels," Hormone Research, vol. 35, no. 3-4, pp. 167-169, 1991.

[24] J. Berwaerts, J. Verhelst, R. Abs, B. Appel, and C. Mahler, "A giant prolactinoma presenting with unilateral exophthalmos: effect of cabergoline and review of the literature," Journal of Endocrinological Investigation, vol. 23, no. 6, pp. 393-398, 2000.

[25] E. Espinosa, E. Sosa, V. Mendoza, C. Ramírez, V. Melgar, and M. Mercado, "Giant prolactinomas: are they really different from ordinary macroprolactinomas?” Endocrine, vol. 52, no. 3, pp. 652-659, 2016.

[26] M. F. Fraioli, F. Novegno, E. Catena, C. Fraioli, and L. Moschettoni, "Multidisciplinary treatment of giant invasive prolactinomas in paediatric age: long-term follow-up in two children," Child's Nervous System, vol. 26, no. 9, pp. 1233-1237, 2010.

[27] V. Fernandes, M. J. Santos, R. Almeida, and O. Marques, “Tenyear follow-up of a giant prolactinoma," BMJ Case Reports, 2015.

[28] D. K. Hamilton, M. L. Vance, P. T. Boulos, and E. R. Laws, "Surgical outcomes in hyporesponsive prolactinomas: analysis of patients with resistance or intolerance to dopamine agonists," Pituitary, vol. 8, no. 1, pp. 53-60, 2005.
[29] V. Primeau, C. Raftopoulos, and D. Maiter, "Outcomes of transsphenoidal surgery in prolactinomas: improvement of hormonal control in dopamine agonist-resistant patients," European Journal of Endocrinology, vol. 166, no. 5, pp. 779-786, 2012.

[30] W. Kim, C. Clelland, I. Yang, and N. Pouratian, "Comprehensive review of stereotactic radiosurgery for medically and surgically refractory pituitary adenomas," Surgical Neurology International, vol. 3, supplement 2, pp. S79-S89, 2012.

[31] E. Delgrange, T. Daems, J. Verhelst, R. Abs, and D. Maiter, "Characterization of resistance to the prolactin-lowering effects of cabergoline in macroprolactinomas: a study in 122 patients," European Journal of Endocrinology, vol. 160, no. 5, pp. 747-752, 2009.

[32] E. Delgrange, J. Trouillas, D. Maiter, J. Donckier, and J. Tourniaire, "Sex-related difference in the growth of prolactinomas: A Clinical and Proliferation Marker Study," Journal of Clinical Endocrinology and Metabolism, vol. 82, no. 7, pp. 2102-2107, 1997.

[33] E. Delgrange, T. Duprez, and D. Maiter, "Influence of parasellar extension of macroprolactinomas defined by magnetic resonance imaging on their responsiveness to dopamine agonist therapy," Clinical Endocrinology, vol. 64, no. 4, pp. 456-462, 2006.

[34] S. Salenave, D. Ancelle, T. Bahougne et al., "Macroprolactinomas in children and adolescents: factors associated with the response to treatment in 77 patients," Journal of Clinical Endocrinology and Metabolism, vol. 100, no. 3, pp. 1177-1186, 2015.

[35] J. Verhelst, R. Abs, D. Maiter et al., "Cabergoline in the treatment of hyperprolactinemia: a study in 455 patients," Journal of Clinical Endocrinology and Metabolism, vol. 84, no. 7, pp. 25182522, 1999. 


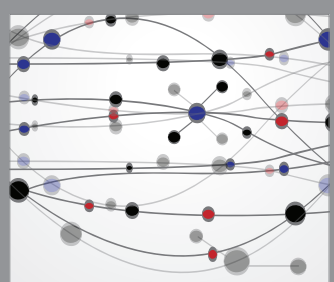

The Scientific World Journal
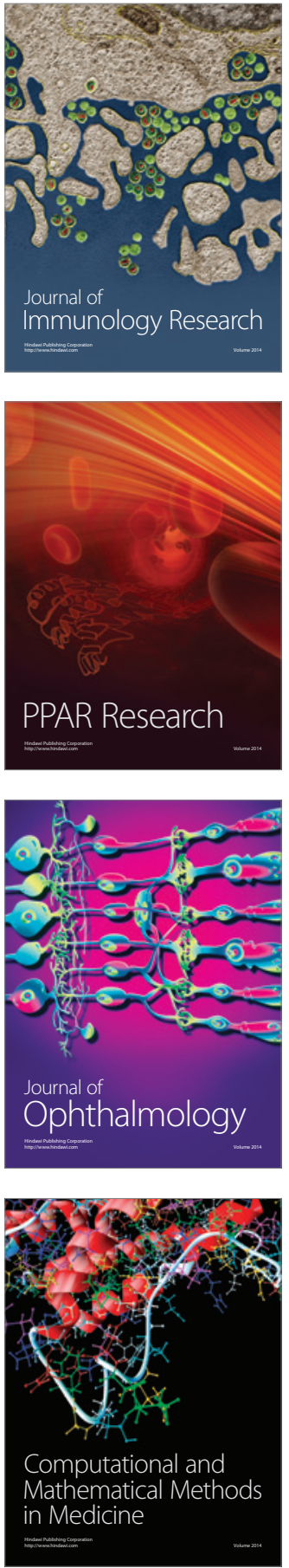

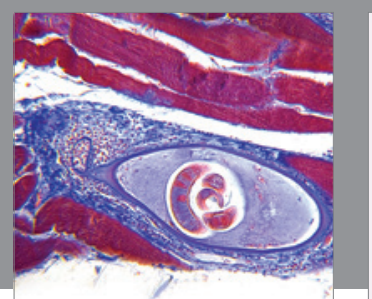

Gastroenterology Research and Practice

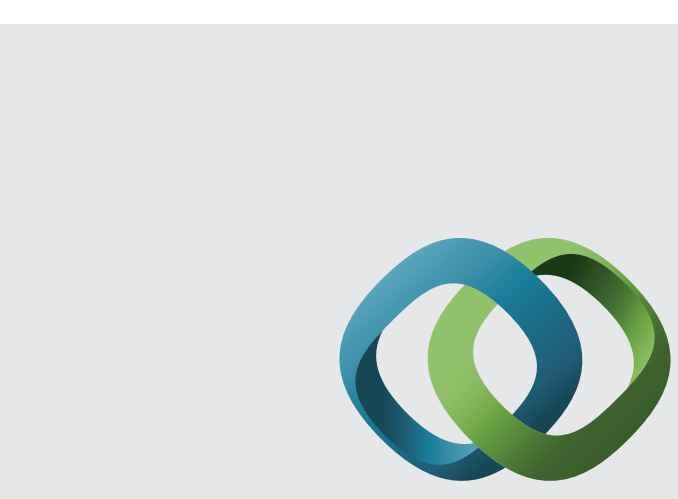

\section{Hindawi}

Submit your manuscripts at

http://www.hindawi.com
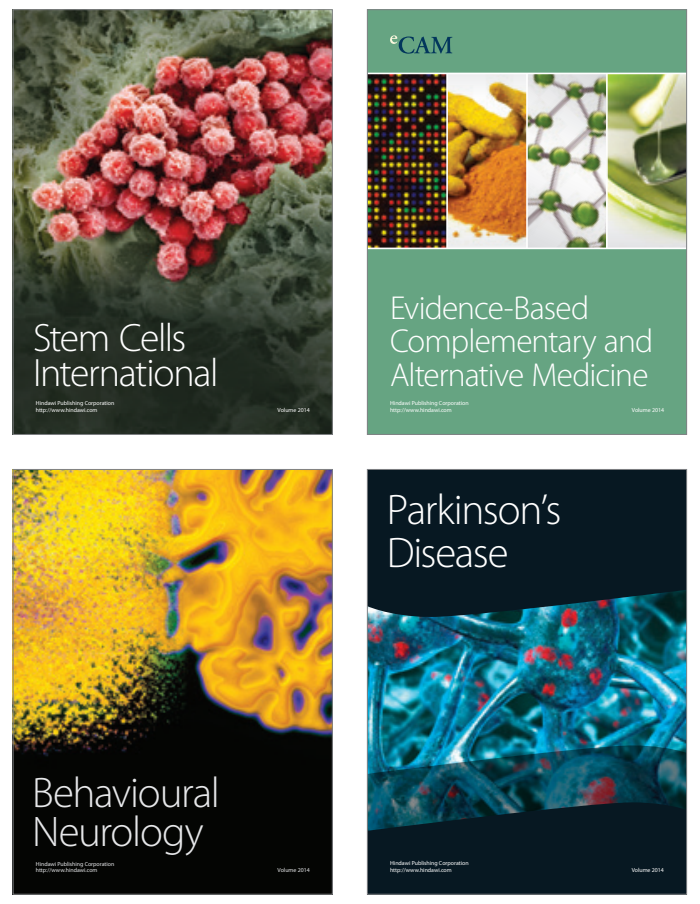
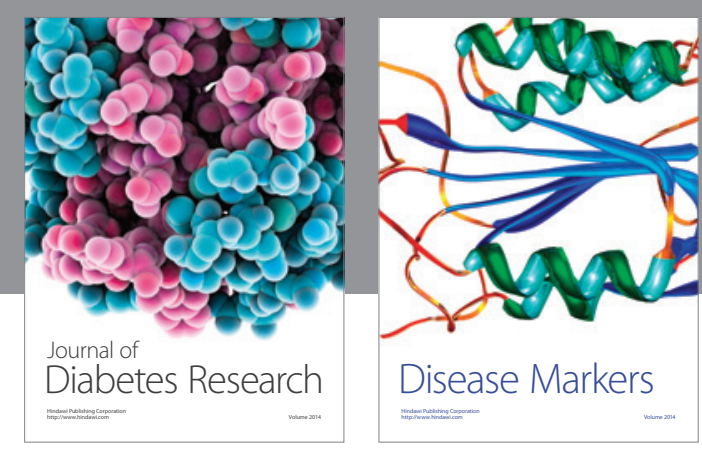

Disease Markers
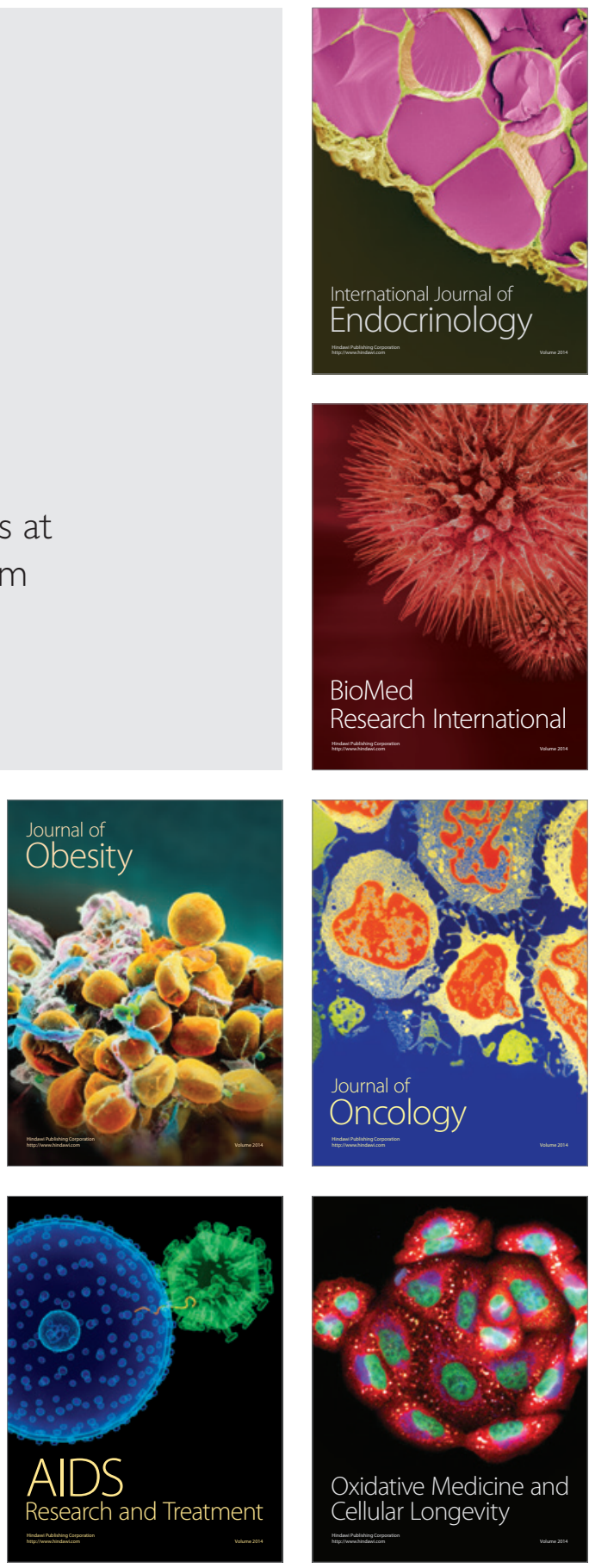